\title{
Hydration Dynamics in Solutions of Cyclic Polyhydroxyl Osmolytes
}

Nimesh Shuklat, Julia Goeks", Erika A. Taylor§ and Christina M. Othon**

$\dagger$ Department of Physics, Wesleyan University, Middletown CT 06457 USA

$\S$ Department of Chemistry, Wesleyan University, Middletown CT 06457 USA

$\$$ Department of Physics, Ripon College, Ripon WI 54971 USA

*FA_Corresponding_Author_Footnote: Christina M. OthonDepartment of Physics, Ripon College, Ripon WI 54971 USA; othonc@ripon.edu

\section{SUPPLEMENTARY MATERIAL}

Absorption spectra were recorded on a spectrophotometer (Beckman-650). The emission spectra were recorded on a spectrometer (Ocean Optics-QE65000). The absorption/emission maximum of LYen in pure water was measured as $426.1 \pm 0.5 \mathrm{~nm}$ spectra and $540.9 \pm 0.5 \mathrm{~nm}$ respectively. The absorption/emission maxima for concentrations of $0.5 \mathrm{M}$ for all three sugar co-solutes were measured to be the same, to within as displayed in Figure S1. Solid lines represent the absorption spectra and dashed lines represent the fluorescence emission spectra. 


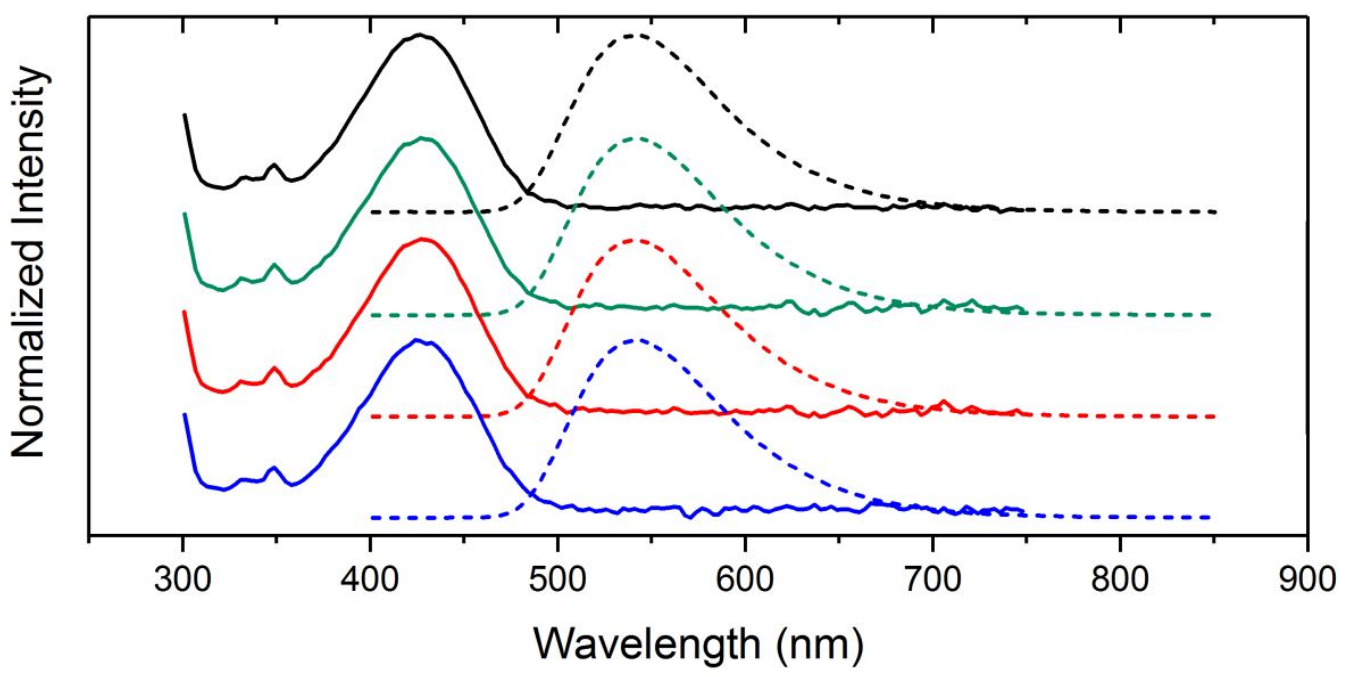

Figure S1. Absorption spectra (solid lines) emission Spectrum (dashed lines). Spectrum for 200 $\mu \mathrm{M}$ LYen in water (black), 0.5 M glucose (green), 0.5 M Me-glucose (red), 0.5 M Myo-inositol (blue).

Lifetime measurements conducted on a time correlated single photon counting apparatus, the output of a high energy oscillator (Coherent Chameleon Ultra II) was passed through a pulse selector (Conoptics) to provide $425 \mathrm{~nm}$ pulses at $10.6 \mathrm{MHz}$ repetition rate. The light was attenuated (to $\sim 1.5 \mathrm{mw}$ ) and vertically polarized by using a half wave plate and polarizer. The light was frequency doubled for excitation of the sample which was placed in a $1 \mathrm{~cm}$ path length quartz cuvette. The residual infrared light was used to generate the synchronization with a fast photodiode whose output was fed to a single photon counting (TCSPC) module (Becker-Hick1 SPC-130 TCSPC). The fluorescence signal was collected at right angle geometry and passed through a precision linear polarizer oriented at the magic angle in order to isolate the effect of anisotropy from lifetime measurements. The collected light was focused onto the input slit of a Jerrell Ash (82-410) monochromator which was then focused onto an avalanche photodiode (id100 ID Quantique). The lifetime of LYen in pure water was measured as $5.85 \pm 0.02 \mathrm{~ns}$. The lifetime of LYen was recorded for sugar concentrations of $0.5 \mathrm{M}$. For Myo-inositol the lifetime was indistinguishable from that of pure water at $5.87 \mathrm{~ns}$. For glucose and $\mathrm{Me}$-Glucose the lifetime increased slightly to 5.98 and 6.18 ns respectively (see Figure S2). 


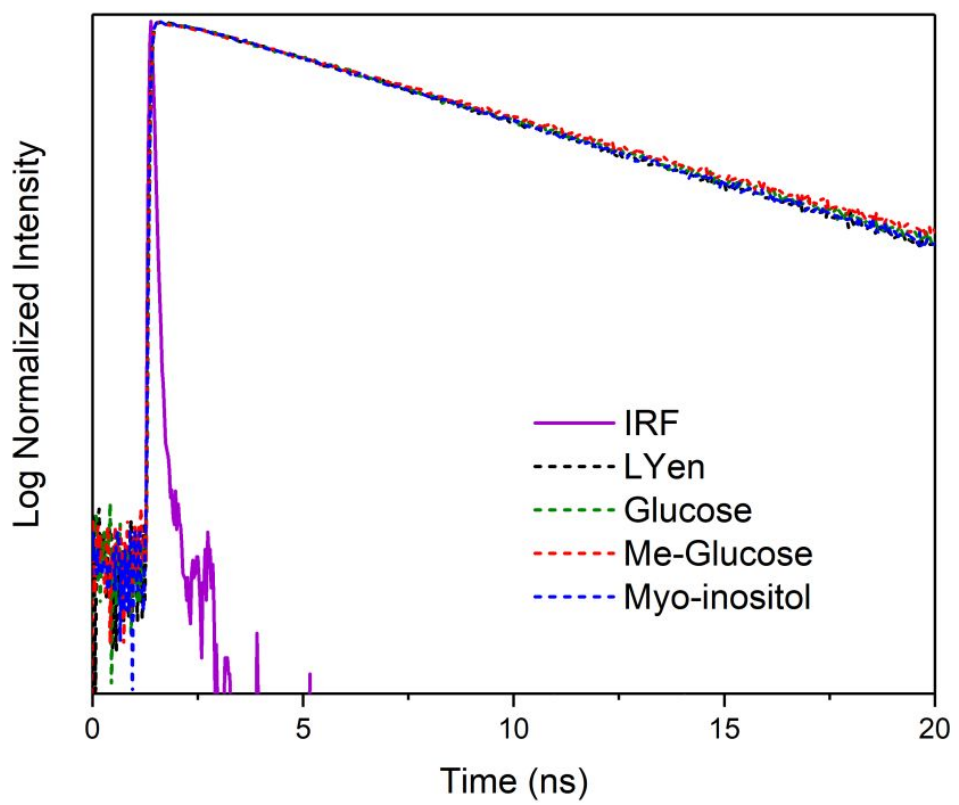

Figure S2. Lifetime of LYen as measured by Time-Correlated Single Photon Counting. Measurements are made on a $200 \mu \mathrm{M}$ LYen sample in water (black) $0.5 \mathrm{M}$ glucose (green), 0.5 M Me-glucose (red), 0.5 M Myo-inositol (blue).

The transients at each wavelength were normalized and fit using a global fit procedure. Typically, the fit function should be a convolution of the instrument response function with the sum of exponential functions. The global fit procedure was implemented using the program Origin. ${ }^{1}$ For fitting an early rise, a Gaussian function convoluted with the Gaussian IRF was used. The analytical expressions used in the global fit are:

$$
\begin{gathered}
D_{I \otimes G}(\lambda, t)=\frac{a_{0}(\lambda)}{2 \sqrt{1+\frac{w^{2}}{\tau_{0}^{2}}}} \exp \left(\frac{\left(t-t_{0}\right)}{\sqrt{1+\frac{w^{2}}{\tau_{0}^{2}}}}\right)^{2}{ }^{2}\left[1+\operatorname{erf}\left(\frac{t-t_{0}}{w \sqrt{1+\frac{w^{2}}{\tau_{0}^{2}}}}\right)\right] \\
D_{I \otimes E, i}(\lambda, t)=\frac{a_{i}(\lambda)}{2} \exp \left(-\frac{t-t_{0}}{\tau_{i}}\right) \exp \left(-\frac{w^{2}}{4 \tau_{i}^{2}}\right)\left[1+\operatorname{erf}\left(\frac{t-t_{0}-\frac{w^{2}}{2 \tau_{i}}}{w}\right)\right]
\end{gathered}
$$




$$
D(\lambda, t)=D_{I \otimes G}(\lambda, t)+\sum_{i=1}^{n} D_{I \otimes E, i}(\lambda, t)
$$

Where $t_{0}$ is the time center, $w$ is the width of the Gaussian IRF, $a_{\mathrm{i}}$ and $\tau_{\mathrm{i}}$ are the amplitude and time constant of the $\mathrm{i}^{\text {th }}$ exponential function and $a_{0}$ and $\tau_{0}$ are the amplitude and the width of the Gaussian function. The above-mentioned function was implemented in Gaussian using a user defined function implemented in Origin using the fitting function organizer. While performing the fit, all the time constants and IRF width was shared and the time constant for lifetime $\tau_{3}$ was kept fixed to the value obtained from independent TCSPC measurements. An orthogonal distance regression method was used in all fitting procedures. For calculating the first moment fitted curves were normalized to the intensity of steady state spectrum and time resolved emission spectrum was constructed by sampling transients at several time intervals for all wavelengths as described in.$^{2,3}$ An example of the quality of the global fitting is shown in Figure $\mathrm{S} 3$ for $0.1 \mathrm{M}$ of concentration of Glucose at wavelengths across the spectrum, the quality of fit is representative of that observed for all of the samples examined in this study. There are four time constants that are fit globally across the spectrum corresponding to the Gaussian convolution of the IRF $\left(\tau_{0}\right)$, two relaxation modes corresponding to vibrational/librational losses $\left(\tau_{1}\right)$ and solvation relaxation $\left(\tau_{2}\right)$, and finally a lifetime component that is fixed and measured independently via time-correlated single photon counting (Figure S2).

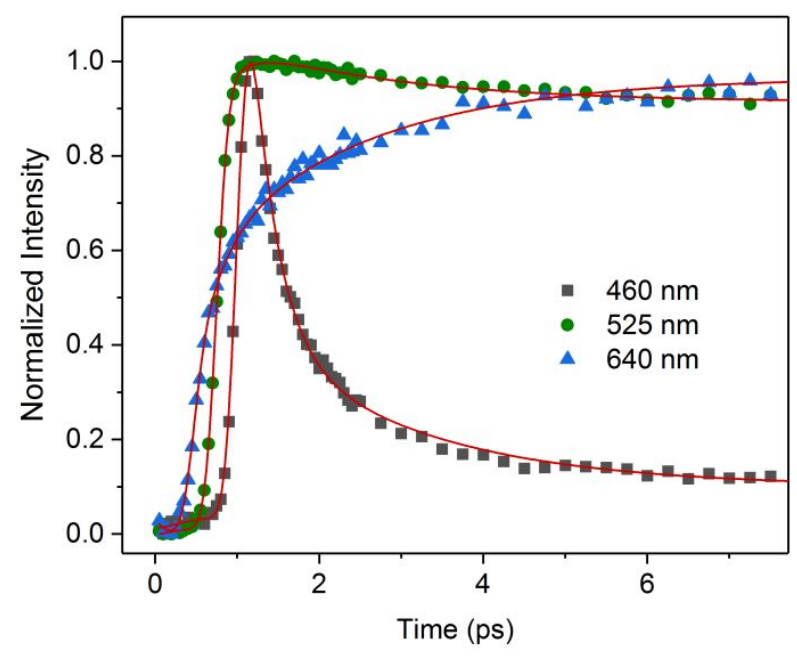

Figure S3. Results of global fitting procedure for a sample of $200 \mu \mathrm{m}$ in a $0.1 \mathrm{M}$ glucose solution for wavelengths sampled across the spectrum. 
Global fitting amplitudes for time-resolved fluorescence frequency spectrum for Lucifer Yellow in $0.5 \mathrm{M}$ concentrations of glucose, $\mathrm{Myo}$-inositol, and $\mathrm{Me}$-Glucose solutions. The data points represent the contribution for the Amplitudes for global fit time-constants: a0 green circles, a1 blue squares, a2 red diamonds, a3 black circles are the amplitudes of Gaussian Instrumental Response Function, fs energy loss, ps solvation relaxation and ns lifetime time constants respectively. Data indicates no significant differences in the relative contributions of relaxation pathways to the spectral relaxation. 


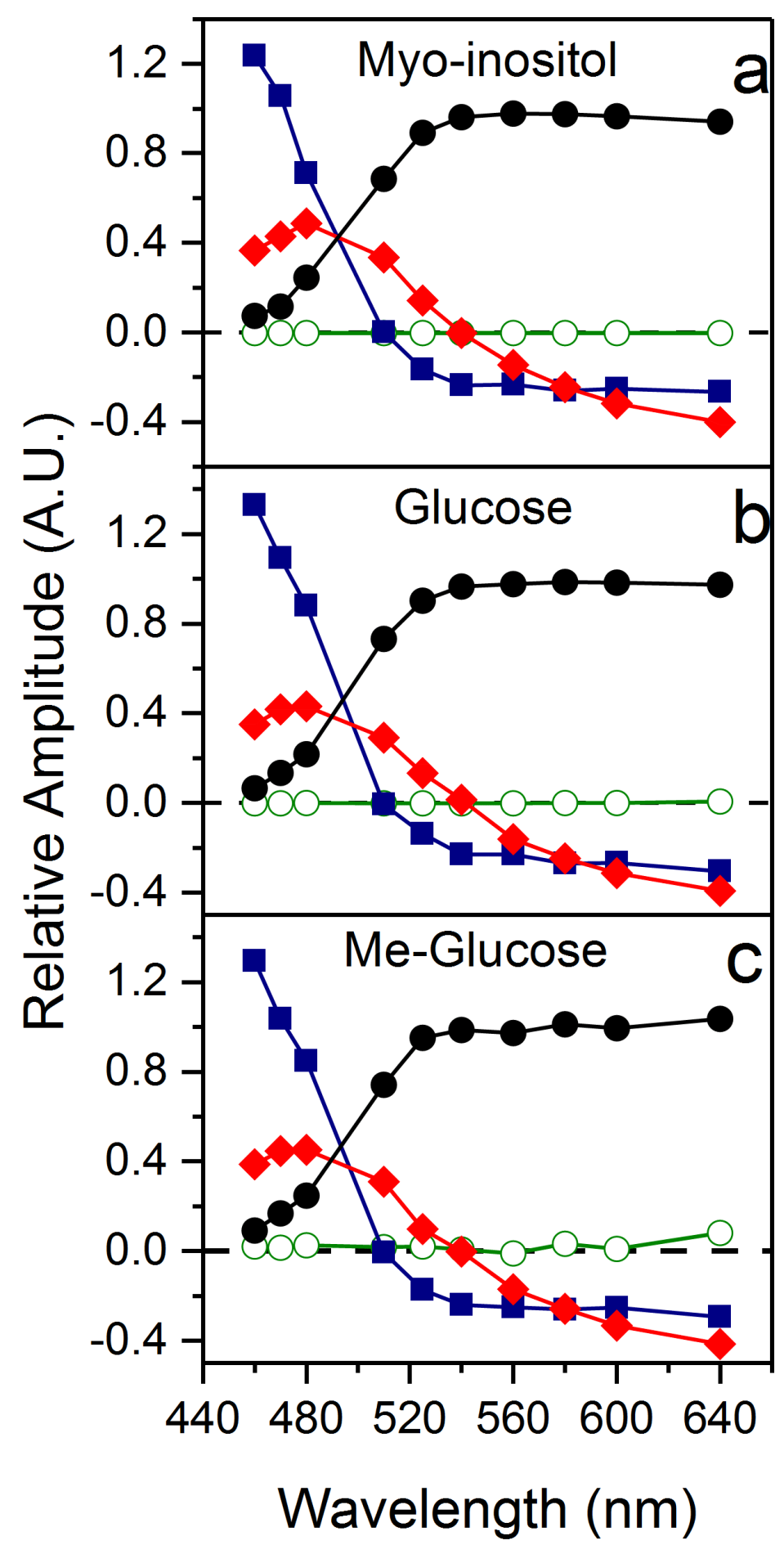

Figure S4. Global fitting amplitudes for time-resolved fluorescence frequency spectrum for Lucifer Yellow in $0.5 \mathrm{M}$ concentrations of (a) glucose, (b) Myo-inositol, and (c) Me-Glucose solutions. The data points represent the contribution for the Amplitudes for global fit timeconstants: a0 green circles, a1 blue squares, a2 red diamonds, a3 black. 
(1) Originlab Corporation https://www.originlab.com/ (accessed Jan 6, 2019).

(2) Lang, B.; Angulo, G.; Vauthey, E. Ultrafast Solvation Dynamics of Coumarin 153 in Imidazolium-Based Ionic Liquids. J. Phys. Chem. A 2006, 110 (22), 7028-7034. https://doi.org/10.1021/jp057482r.

(3) Alexandre, F.; Vauthey, E. Ultrafast Excited-State Dynamics of Oxazole Yellow DNA Intercalators. J. Phys. Chem. B 2007, 111 (43), 12610-12620. https://doi.org/10.1021/JP073182T. 